\title{
ReDSHIFT: A Global Approach to Space Debris Mitigation
}

\author{
Alessandro Rossi ${ }^{1, *(D)}$, Camilla Colombo ${ }^{2}$, Kleomenis Tsiganis ${ }^{3}$ (D), James Beck ${ }^{4}$, \\ Jonathan Becedas Rodriguez $^{5}$, Scott Walker ${ }^{6}$, Federico Letterio ${ }^{7}$ (D), Florio Dalla Vedova ${ }^{8}$, \\ Volker Schaus 9 (iD, Rada Popova ${ }^{10}$, Alessandro Francesconi ${ }^{11}$, Hedley Stokes ${ }^{12}$, \\ Thorn Schleutker $^{13}$ (D), Elisa Maria Alessi ${ }^{1}$ (D), Giulia Schettino ${ }^{1}$ (D), Ioannis Gkolias ${ }^{2}$ (D), \\ Despoina K. Skoulidou ${ }^{3}$, Ian Holbrough ${ }^{4}$, Franco Bernelli Zazzera ${ }^{2}$ (D), Enrico Stoll ${ }^{9}$ (D) and \\ Youngkyu Kim 10 \\ 1 IFAC-CNR, 50019 Sesto Fiorentino (FI), Italy; em.alessi@ifac.cnr.it (E.M.A.); g.schettino@ifac.cnr.it (G.S.) \\ 2 Dipartimento di Scienze e Tecnologie Aerospaziali, Politecnico di Milano, 20156 Milano, Italy; \\ camilla.colombo@polimi.it (C.C.); ioannis.gkolias@polimi.it (I.G.); franco.bernelli@polimi.it (F.B.Z.) \\ 3 Department of Physics, Section Astrophysics, Astronomy and Mechanics, Aristotle University, \\ 54006 Thessaloniki, Greece; tsiganis@auth.gr (K.T.); dskoulid@physics.auth.gr (D.K.S.) \\ $4 \quad$ Belstead Research Ltd., Ashford TN25 4PF, UK; james.beck@belstead.com (J.B.); \\ ian.holbrough@belstead.com (I.H.) \\ 5 Elecnor Deimos Satellite Systems, 13500 Puertollano, Spain; jonathan.becedas@deimos-space.com \\ 6 Astronautics Research Group, University of Southampton, Southampton SO17 1BJ, UK; sjiw@soton.ac.uk \\ 7 Deimos Space, Tres Cantos, 28760 Madrid, Spain; federico.letterio@deimos-space.com \\ 8 LuxSpace Sarl, 6832 Betzdorf, Luxemburg; dallavedova@luxspace.lu \\ 9 Institute of Space Systems, Technische Universität Carolo-Wilhemina zu Braunschweig, 38108 Braunschweig, \\ Germany; v.schaus@tu-braunschweig.de (V.S.); e.stoll@tu-braunschweig.de (E.S.) \\ 10 Institute of Air and Space Law, University of Cologne, 50923 Cologne, Germany; \\ rada.popova@gmail.com (R.P.); kim.youngkyu@hotmail.com (Y.K.) \\ 11 Dipartimento di Ingegneria Industriale, University of Padova, 35131 Padova, Italy; \\ alessandro.francesconi@unipd.it \\ 12 PHS Space Ltd., Cerne Abbas DT2 7GW, UK; hedley_stokes@msn.com \\ 13 DLR (German Aerospace Center), 51147 Koeln, Germany; thorn.schleutker@dlr.de \\ * Correspondence: a.rossi@ifac.cnr.it
}

Received: 19 March 2018; Accepted: 6 June 2018; Published: 13 June 2018

\begin{abstract}
The H2020 ReDSHIFT project aims at finding passive means to mitigate the proliferation of space debris. This goal is pursued by a twofold research activity based on theoretical astrodynamics, computer simulations and the analysis of legal aspects of space debris, coupled with an experimental activity on advanced additive manufacturing (3D printing) applied to the production of a novel small satellite. Several different aspects related to the design and production of a debris compliant spacecraft are treated, including shielding, area augmentation devices for deorbiting (solar and drag sails) and design for demise. A strong testing activity, mainly based on design for demise wind tunnel experiments and hypervelocity impacts is performed as well. The main results obtained so far in the project are outlined.
\end{abstract}

Keywords: space debris; mitigation; astrodynamics; 3D printing

\section{Introduction}

The impact of debris on the space activities has to be reduced by adopting a global strategy able to address the problem from different points of view, from the very beginning of the planning of a space mission. As an example, the choice of the orbit, the spacecraft bus and its shielding, the spacecraft 
power system and propulsion, are all aspects that should be optimized, having in mind not only the goal of the mission but also the minimization of the "environmental" impact of the spacecraft. The space debris related aspects can be summarized in terms of: prevention, protection, mitigation and regulation. To tackle these issues, the project called Revolutionary Design of Spacecraft through Holistic Integration of Future Technologies (ReDSHIFT) was funded by the European Union in the framework of the PROTEC Call of Horizon 2020 (see http:/ / redshift-h2020.eu/). With 13 European partners, ReDSHIFT is a very large project gathering results on a wide range of open problems in the realm of the space debris related studies. It is grounded on two main pillars, a theoretical and an experimental one. While these two pillars appear to belong to different areas of interest, it is important to approach both fields in a single project with the goal of finding links and synergies that can enhance the effectiveness of the solutions found in the study. The main purpose of this paper is to briefly describe the main results of the project achieved so far trying, where possible, to highlight the connections between the theoretical findings and the experimental achievements.

The theoretical part of ReDSHIFT is mainly based on a complete mapping of the Low Earth Orbit (LEO) to Geostationary Orbit (GEO) space looking for "dynamical" disposal strategies for any orbital regime, exploiting natural perturbations to spacecraft orbits. Some preferential deorbiting routes, dubbed "deorbiting highways", result from specific resonances populating the different orbital regimes involving gravitational and non-gravitational perturbations. In order to properly exploit the solar radiation pressure driven resonances, the use of area augmentation devices is studied in detail. The main results on this subject are outlined in Section 2.

Furthermore, an extensive campaign of long term simulations of the space debris environment was performed (see [1]) with a twofold aim. First, to identify and quantify the benefits of the proposed solutions. Second, to characterize the particle flux impacting on a spacecraft under different future scenarios with the aim of evaluating the effectiveness of the shields designed in the experimental part.

From the astrodynamics results, several engineering requirements are derived for the area augmentation devices to be included in the spacecraft design, with the aim of achieving an effective deorbiting of the satellite at the end-of-life. The compatibility of these devices with the 3D printed spacecraft (described in Section 3) is also analyzed within the project. Moreover, the re-entry trajectories of the objects deorbited through the "deorbiting highways" (both with and without the area augmentation devices) are studied to characterise the behaviour of the spacecraft in terms of Design for Demise (D4D) when interacting with the atmosphere. These information will, in turn, be transferred to the design phase to identify materials and 3D printed structures that are not going to survive the re-entry phase.

The experimental pillar is mainly devoted to the design and production of a novel spacecraft by $3 \mathrm{D}$ printing. In fact, in an effort to make novel technological solutions easier and more attractive to produce and to implement into future design, thus enhancing the debris-oriented vision of spacecraft manufacturing, ReDSHIFT will explore the possibility to use the now blossoming additive manufacturing technology, to actually realize a model spacecraft and, in particular, some specific parts related to the debris mitigation issues, such as, e.g., the shielding, a sail canister, the sail hatches and joints, etc. As mentioned above, the characteristics of the sail will also be dictated by the needs emerging from the dynamical studies. Innovative shielding against small particles was studied and shall be directly embedded in the aluminum printed spacecraft structure. The materials and components of the spacecraft were tested for D4D in a heated wind tunnel. The D4D tests also included an unprecedented test on an engineering model of a reaction wheel. The first prototype spacecraft (an 8-unit CubeSat) is now in the production phase and will be tested (including launch vibration tests) in the late spring. Based on the results of the first testing phase an improved design, expressly tailored to the 3D printing peculiarities, will be performed and the final ReDSHIFT prototype will be printed in the second half of 2018. The final 3D printed spacecraft will be a fully tested prototype of a small satellite, expressly designed having in mind the space debris issues and regulations. From this prototype a fully functional specimen could be produced with limited additional effort, paving the way to significant innovation 
in the field. The main results of the experimental branch of the project are outlined in Section 3. The results of the dynamical mapping and the demisability analysis are then integrated into a software tool that shall help the users to conceive a "debris compliant" space mission from the design to the disposal phase encompassing all the above findings. This powerful tool is briefly described in Section 4 . Finally, the possible improvements to the international space regulations and standards, stemming from the project's findings, are analyzed and outlined in Section 5.

As already mentioned above, it is worth stressing again that this paper is presenting the main results of ongoing research activities within a project encompassing 13 research units. The ambition of ReDSHIFT, and the common thread linking the following sections, is the attempt to approach the debris mitigation issues from different perspectives, prone to suggest innovative solutions to this pressing problem.

\section{Dynamical Mapping}

As also shown by the long term simulation of the environment performed within the project [1], the disposal of objects after the end-of-life is one of the most important mitigation measures needed to reduce the growth of space debris in the forthcoming years. In order to facilitate this action it is important to identify stable and unstable regions in the phase space where the objects could be moved to exploit either long term "graveyards" or, possibly and preferentially, faster escape routes. To this purpose, a dynamical mapping of the circumterrestrial space was performed within ReDSHIFT.

The whole space, from LEO up to the geostationary orbit, was divided in a fine grid in the orbital elements space. Figure 1 shows a sample of the LEO grid. It highlights the fact that the grids are not uniform and are made denser were the population of objects is more abundant. About 20 million of the initial conditions were propagated, using three different orbital propagators, having similar dynamical models expressly tailored to the explored orbital regime, but different underlying computational engines.

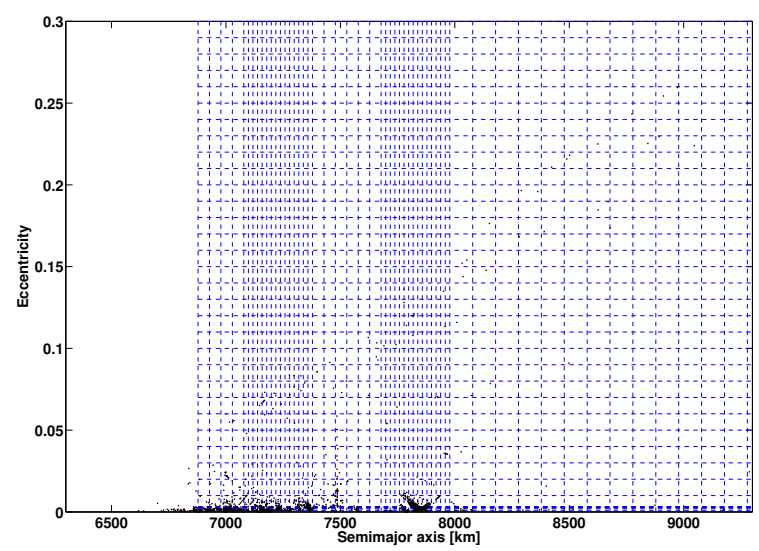

Figure 1. Example of the grid adopted in semimajor axis and eccentricity for the LEO region. The black dots represent the location of the satellites currently in space according to MASTER 2009.

All the orbital propagations were repeated twice: once assuming for the test object an area-to mass ratio, $A / \mathrm{m}$ equal to the standard satellites $\left(0.012 \mathrm{~m}^{2} / \mathrm{kg}\right)$ and then assuming an augmented $A / \mathrm{m}$ $\left(1 \mathrm{~m}^{2} / \mathrm{kg}\right.$, thus simulating the presence of some sail). In both cases the reflectivity coefficient $c_{R}$ was considered equal to 1 and the drag coefficient $c_{D}$ equal to 2.1. The output of these massive orbital propagations was stored in terms of maps displaying, e.g., the lifetime or the maximum eccentricity growth for all the orbits during the 120 year time span of the propagation (the so-called Maximum Eccentricity Maps). Examples of the maps obtained, for the different orbital regimes are shown in Figures 2-5. All the maps produced can be found in the website of the project (http: \redshift-h2020.eu) 
and the theoretical analysis of the results obtained in the mapping can be found in [2-6] and cannot be repeated here for lack of space.

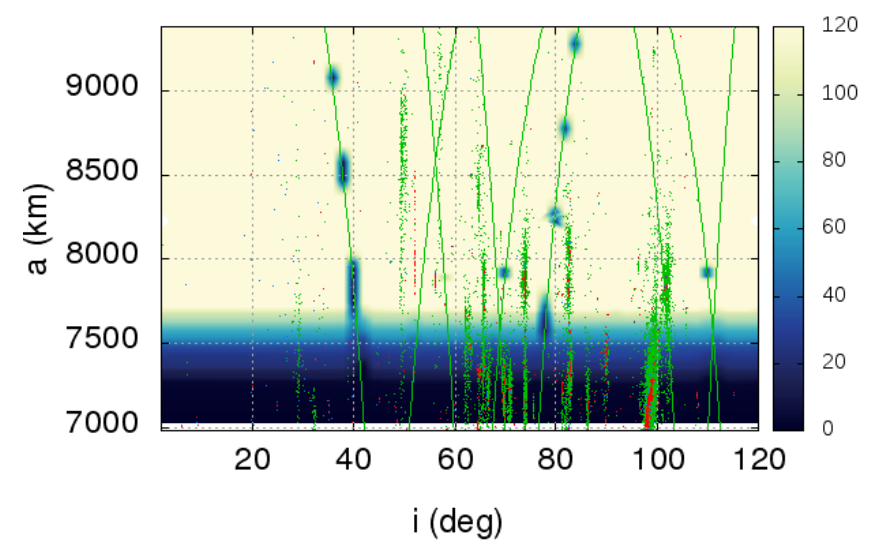

Figure 2. Location of the dominant resonances determining the long-term behavior in LEO (green lines). The color map shows the residual lifetime, as a function of the initial inclination and semi-major axis, computed in 120 years for an object with $A / m=1 \mathrm{~m}^{2} / \mathrm{kg}$, starting from $e=0.001, \Omega=0^{\circ}$ and $\omega=0^{\circ}$. The red and green dots show the location of the spacecraft and fragments, respectively, according to MASTER2009.

Figure 2 shows the location of the resonances determining the long-term behavior in LEO [7], superimposed on a residual lifetime map. The objects considered start from initially circular orbits and have an area over mass ratio $A / m=1 \mathrm{~m}^{2} / \mathrm{kg}$. The dark blue regions at the bottom of Figure 2 correspond to residual lifetimes compliant with the 25-year rule [8]. The small green and red dots show the location of all the objects present in the MASTER 2009 population.

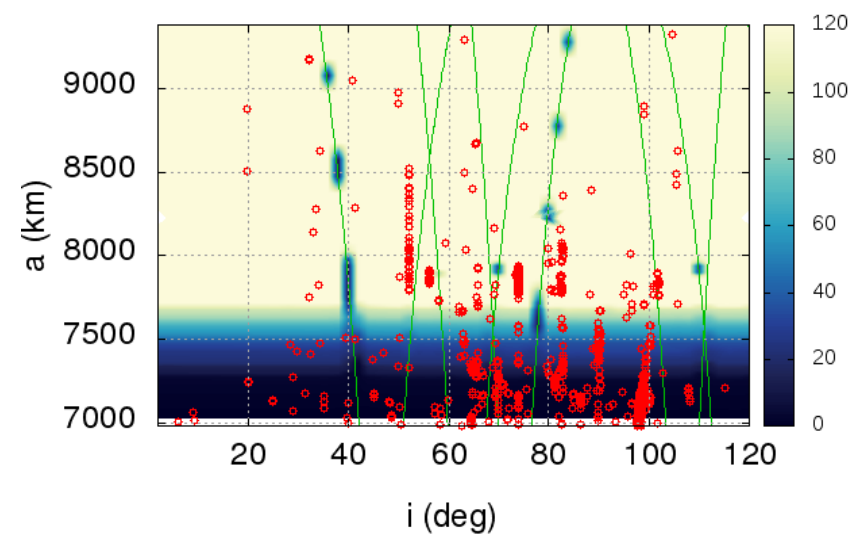

Figure 3. The same as Figure 2, but showing, with small red circles, only the location of the satellites (active and non active), according to MASTER2009.

Similarly, Figure 3 shows the same map but with only the satellites now highlighted with red circles. It can be noticed how many of the satellites now in space lie in orbits quite close (in terms of orbital elements) to some of the resonances. It could be argued that some specific missions, populating the long lifetime yellowish regions, might be preferentially steered, already in the design phase, towards the resonance spots, in order to benefit from the augmented disposal speed granted by the unstable resonant orbits, saving the propellant needed to move the satellite toward the entry into the resonance, at the end-of-life. Of course such steering should be leveraged with the mission purposes and might sometime be unfeasible. Nonetheless, this issue will be further explored also in the framework of the legal aspects of the project (see Section 5). On the same line, maximum eccentricity 
maps were produced for the Medium Earth Orbits (MEO) and the main resonances in that region were highlighted [4]. Figure 4 shows that there exists indeed the possibility to deorbit from the Global Navigation Satellite Systems (GNSS) altitude by exploiting the natural growth of eccentricity of the unstable orbits. Whereas the time required for this deorbiting exceeds the classical 25-year timespan, it is worth stressing that the actual time spent in the LEO protected region by a spacecraft re-entering from MEO in an elliptical trajectory is just a small fraction of its residual lifetime.

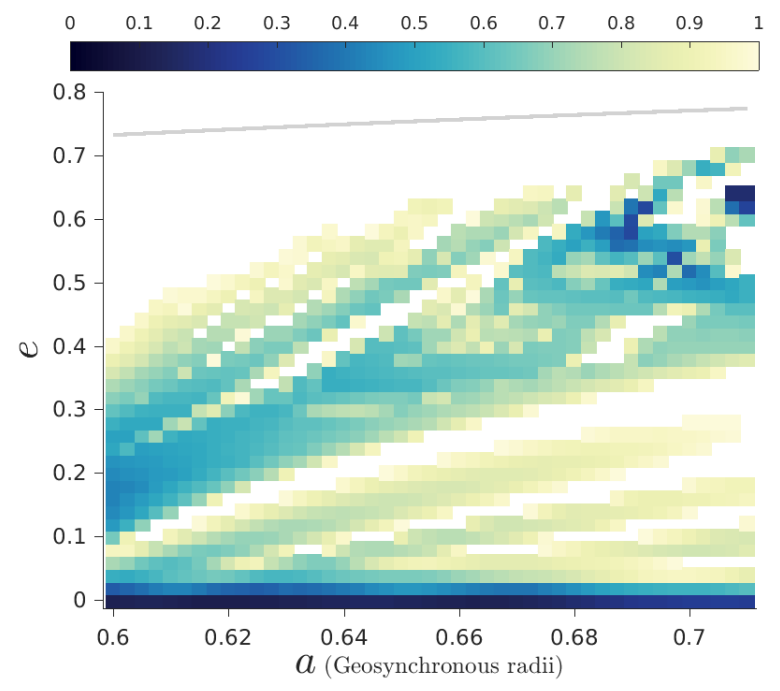

Figure 4. Maximum eccentricity map for circular medium Earth orbits with inclination $i=56^{\circ}$, in the GNSS region. The white regions indicate that the eccentricity needed to re-enter into the atmosphere is reached within 120 years. An $A / m=1 \mathrm{~m}^{2} / \mathrm{kg}$ is assumed.

For the GEO region, both the geosynchronous equatorial orbits and the highly inclined geosynchronous orbits (e.g., used recently by the Chinese Compass system) were explored. In the former case, whereas natural re-entry solutions cannot be found, stable graveyard orbits were identified. The map in Figure 5 (left panel) shows the maximum variation in semimajor axis of the graveyard orbits around the geostationary altitude. The right panel of Figure 5 alternatively shows the maximum eccentricity variations reached by a spacecraft starting from an inclined GEO, mainly due to lunar perturbations [3]. A significant increase in the orbital eccentricity can be noticed for these cases, paving the way to possible disposal manoeuvres, even from these high Earth orbits.
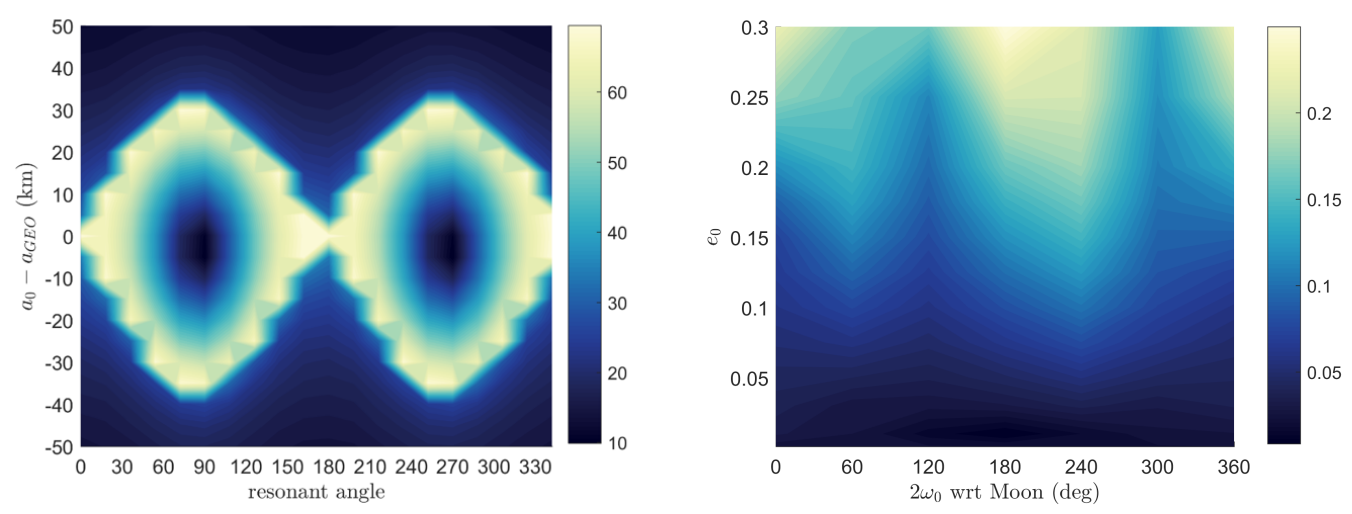

Figure 5. Left panel: Variation of $\Delta a$ over 30 years. Initial orbit with $a_{G E O}+[-50 ;+50] \mathrm{km}, e_{0}=0.01$, $i_{0}=0^{\circ}$ and $\lambda_{0}=[0,360]$ deg. Right panel: Effect of the luni-solar perturbations for highly inclined initial GEO orbits (initial inclination equal to $55^{\circ}$ ). 
As the next step in the project, the end-of-life disposal manoeuvres needed to exploit the natural dynamics, revealed through the long-term orbit evolution maps, the analytical resonance study and the frequency analysis briefly described above, were computed (e.g., [9] for the LEO part). The main novelty of ReDSHIFT is that the natural dynamics are enhanced via impulsive manoeuvres, solar and drag sailing and a combination of manoeuvre and solar and drag sailing. The end-of-life manoeuvre requirements need to be designed based on mission characteristics such as the available on-board propellant and the area-to-mass ratio of the satellite. Exploiting the developed methods, it is possible to determine the optimal re-entry or graveyard orbit, given an available $\Delta V$ on board the satellite. Slightly different design strategies are followed for the LEO, MEO and GEO environment. The strategy followed for disposal in LEO consists in computing the possible displacements in $(a, e, i)$ for a given $\Delta V$ provided in input, in order to identify the most suitable final orbit that can be achieved with that $\Delta V$. The maximum $\Delta V$ considered for the simulation is the one corresponding to a direct re-entry manoeuvre down to $80 \mathrm{~km}$ of altitude. The maximum value of $\Delta V$ considered for the simulation is the one corresponding to a direct re-entry manoeuvre down to $80 \mathrm{~km}$ altitude. In this way, all the possible target orbits are selected and, among them, the one that will naturally evolve toward re-entry in a time less than 50 years is chosen. For all these cases, the required $\Delta V$ is computed as a single burn manoeuvre in case the two orbits are intersecting. As an example, Figure 6 shows the solutions for re-entry from low-Earth orbit, the colour bar represents the $\Delta V$ requirements.
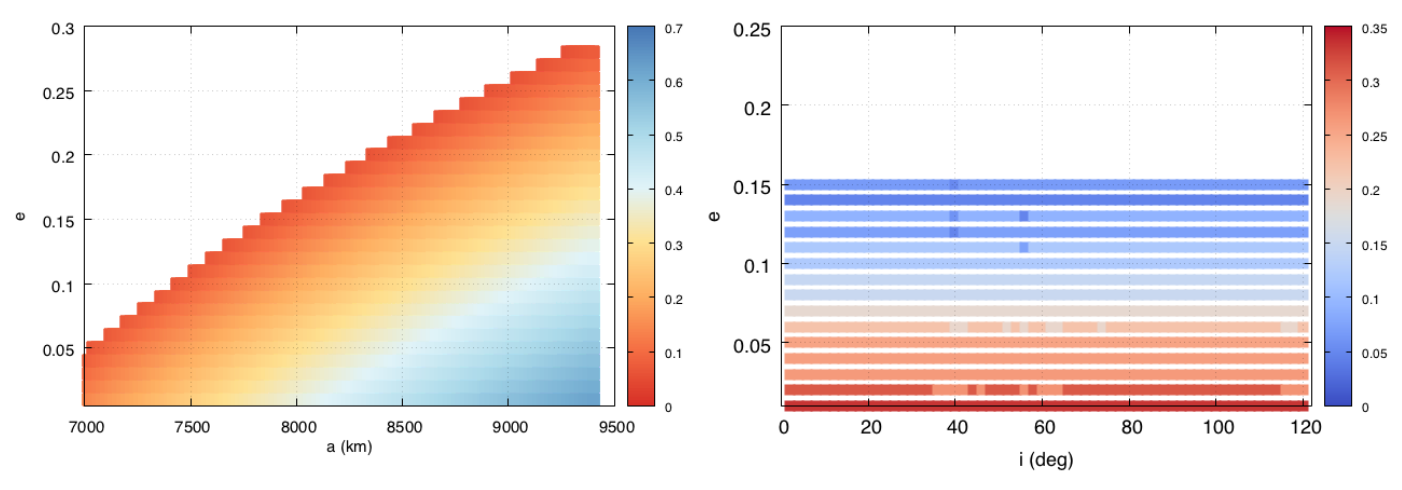

Figure 6. Example of solutions for re-entry from low-Earth orbit, the colour bar represents the $\Delta V$ requirements, in $\mathrm{km} / \mathrm{s}$. Left panel: results for the direct re-entry solutions. Right panel: minimum-cost re-entry solutions, $a_{0}=R_{E}+1520 \mathrm{~km}$.

Moreover, as mentioned above, a solar radiation pressure-augmented case, considering an $\mathrm{A} / \mathrm{m}$ of $1 \mathrm{~m}^{2} / \mathrm{kg}$, was studied. In this case the corresponding resonant inclination bands, presented in [2,7], are targeted. This corresponds to a two-phase deorbiting strategy: a relatively small manoeuvre is performed to reach a resonance where the atmospheric drag or the solar radiation pressure can then be exploited to re-enter by means of a passive stabilised sail.

For the MEO and GEO region, a similar design strategy was devised: given the initial post-mission orbit and the maximum available $\Delta V$ on-board the satellite, the reachable orbital element domain is computed via a single manoeuvre. From all the reachable orbits, we exclude orbits in the GEO protected region and a MEO protected region, that was defined in ReDSHIFT around the GNSS satellite constellation. The required manoeuvre sequence to reach each one of this target orbit is computed. In the MEO case, a transfer with 1 and 2-burn manoeuvre given at the characteristic points of the orbit (i.e., apogee, perigee, nodes) is performed. In the GEO case, the optimal transfer between the last operational orbit and any of the possible target orbits is computed with a 2-burn manoeuvre, through the Lambert problem solved on a grid of initial and final transfer times. The best results are chosen by representing the Pareto front solutions in terms of the minimum $\Delta V$ and re-entry time. Figure 7 shows an example of Pareto front solutions for re-entry orbit of graveyard orbit started from a GEO orbit [10]. 
For the conventional GEO ring, graveyard solution exists and their stability can be measured in terms of the maximum variation of eccentricity in 120 years (see Figure 7 left). For higher initial inclinations, instead, re-entry is possible in less than 120 years as it is shown in Pareto front in (see Figure 7 right). The quality of a re-entry solution can be represented in terms of $\Delta V$ to reach the natural re-entry corridor and the re-entry time.
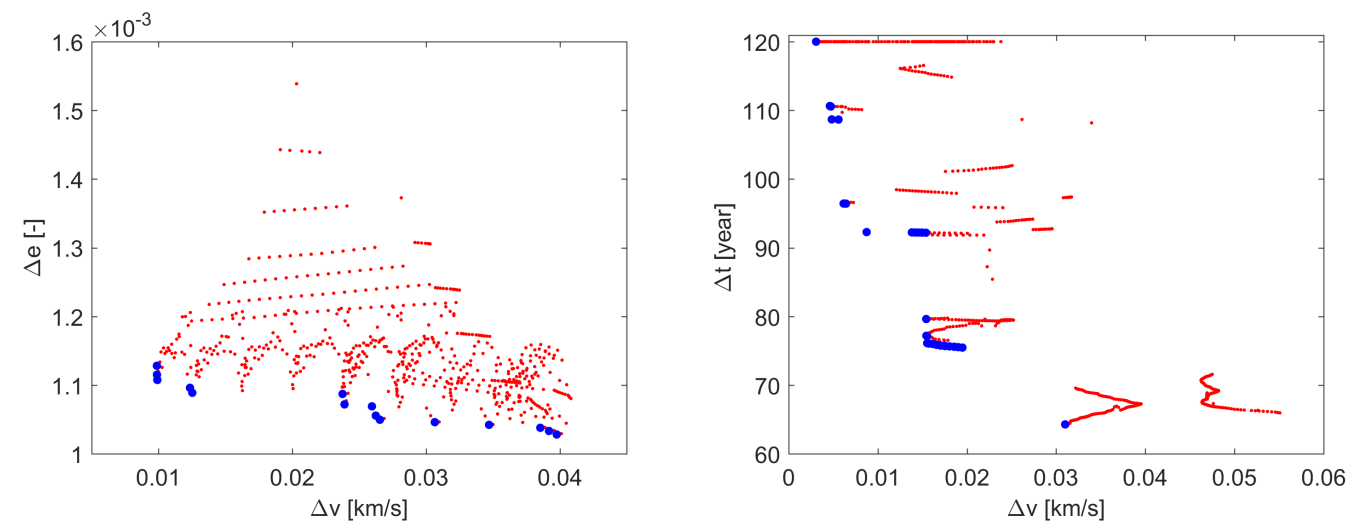

Figure 7. Example of solutions for re-entry and graveyard solution from GEO [10]. Left panel: Pareto front for graveyard solutions for equatorial GEO orbits. Right panel: Pareto front of re-entry solutions from inclined GEO orbit.

An example of the Pareto front solutions for re-entry orbit from MEO is instead shown in Figure 8. For all 1-burn (dark triangles) and 2-burn Hohmann (light grey point) manoeuvres found. The blue/red curve is the Pareto front. A number of possible solutions with $\Delta V \leq 200 \mathrm{~m} / \mathrm{s}$ and $T_{r} \geq 60$ years can be seen.

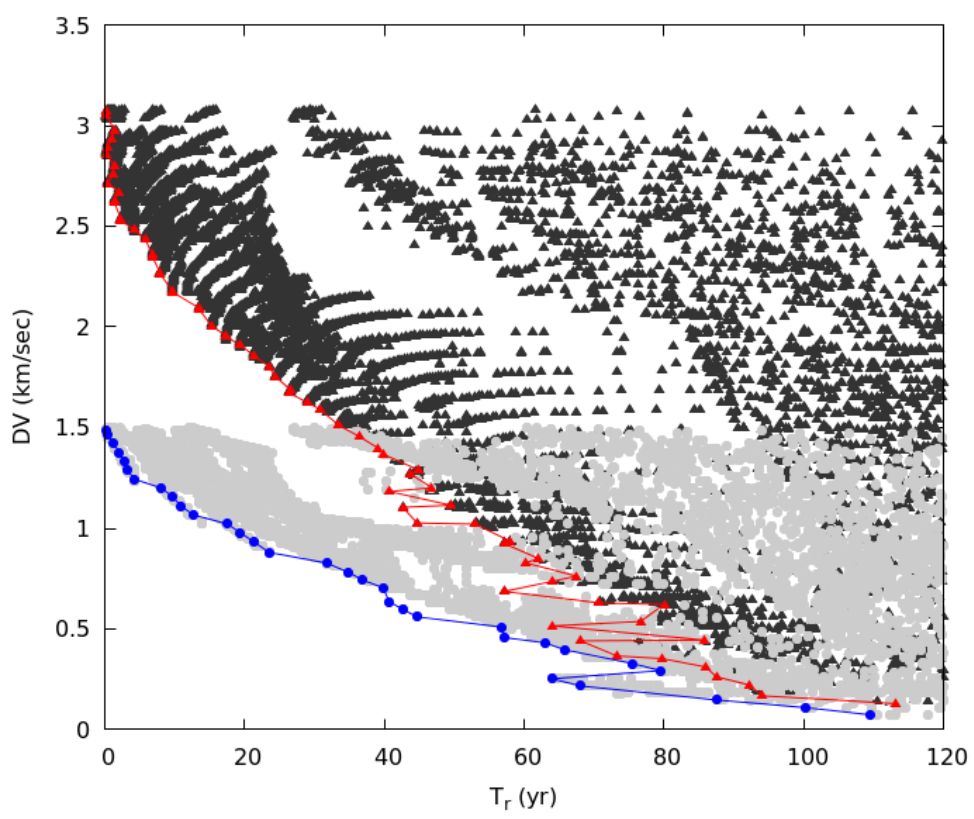

Figure 8. Map of the $\Delta V$ needed to deorbit a typical Galileo spacecraft versus the time $T_{r}$ needed to reach the re-entry for all 1-burn (dark) and 2-burn Hohmann (light grey) maneuvers found. The blue/red curve is the pareto front. A number of possible solutions with $\Delta V \leq 200 \mathrm{~m} / \mathrm{s}$ and $T_{r} \geq 60$ years can be noticed.

Within the ReDSHIFT project, the disposal via solar and drag was studied too, with particular emphasis on the use of solar sailing that allows deorbiting for orbits with perigee higher than 
$600 \mathrm{~km}$. Conventional active solar sailing for deorbiting aims at maximising the cross area of the sail perpendicular to the spacecraft-Sun direction when the spacecraft is moving towards the Sun, while the sail area is minimised when the spacecraft is flying away from the Sun. A second strategy keeps the reflective surface always oriented towards the Sun and controls it through passive stabilisation. The passive and active solar sailing techniques were compared in [11] to determine the most efficient deorbiting strategy under a maximum deorbiting time constraint. An optimisation algorithm was implemented to find the best deorbiting parameter required (i.e., surface area and time to deorbit). Maps of the best deorbiting parameters for active/passive solar sailing were obtained. A third novel strategy for solar sailing has been proposed in the ReDSHIFT project, named as modulating solar sailing. The sail area is oriented perpendicular or at feather with respect to the Sun, with a period of about six months so that the eccentricity of the orbit is constantly increased until re-entry. It has been demonstrated that, with such a sail control, satellites up to the MEO region can be deorbited with a small sail area and a deorbiting time less than 25 years. As an example, Figure 9 shows the sail requirements for deorbiting in 25 years using the modulating solar radiation pressure strategy.

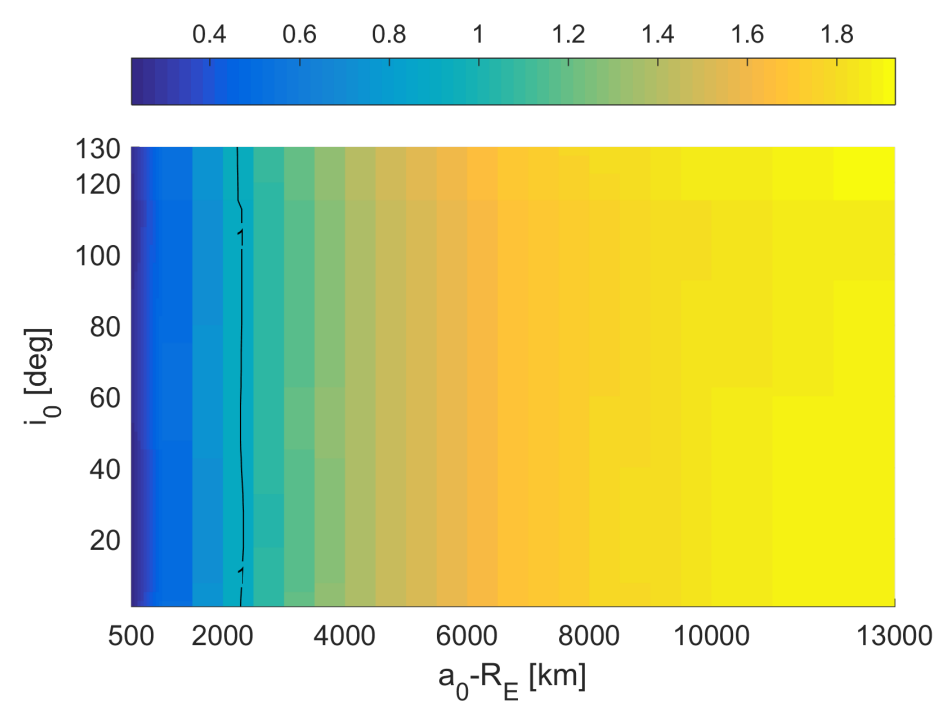

Figure 9. Requirements in terms of effective area-to-mass (i.e., $c_{R} A / m$ ) for different deorbiting times with modulating solar radiation pressure strategy. The colour bar represents the required effective area-to-mass.

\section{Additive Manufacturing}

The starting point for the design of the spacecraft is the definition of the mission requirements. The selected purpose of the spacecraft is an Earth Observation mission as detailed in Table 1.

Table 1. Main features of the selected mission.

\begin{tabular}{cc}
\hline Characteristic & Selected Value \\
\hline Description & Earth observation multi-spectral mission with resolution $39 \mathrm{~m}(\mathrm{GSD})$ \\
Launch mass & $8 \mathrm{~kg}$ \\
Size & $8 \mathrm{U}$ as per CubeSat standard $(200 \times 200 \times 200 \mathrm{~mm})$ \\
Orbit type & $\mathrm{LEO}$ \\
Altitude & $500 \mathrm{~km}$ \\
Inclination & $97.4^{\circ}$ \\
Power & Battery capacity $96 \mathrm{Wh} /$ Power generation: $20 \mathrm{~W}$ (Housekeeping mode) \\
\hline
\end{tabular}


An 8U CubeSat design has been provided by Elecnor Deimos Satellite Systems (EDSS) to act as a baseline configuration to study the potential application of additive manufacturing to small satellite primary structures. Whereas some of the sections of the spacecraft are currently being 3D printed, it is worth stressing that the first detailed design is mostly based on traditional manufacturing techniques (see Figure 10, right panel). After the first round of testing (vibrational, radiation, etc.) the design will be updated and a more advanced exploitation of the additive manufacturing techniques will be explored and applied.
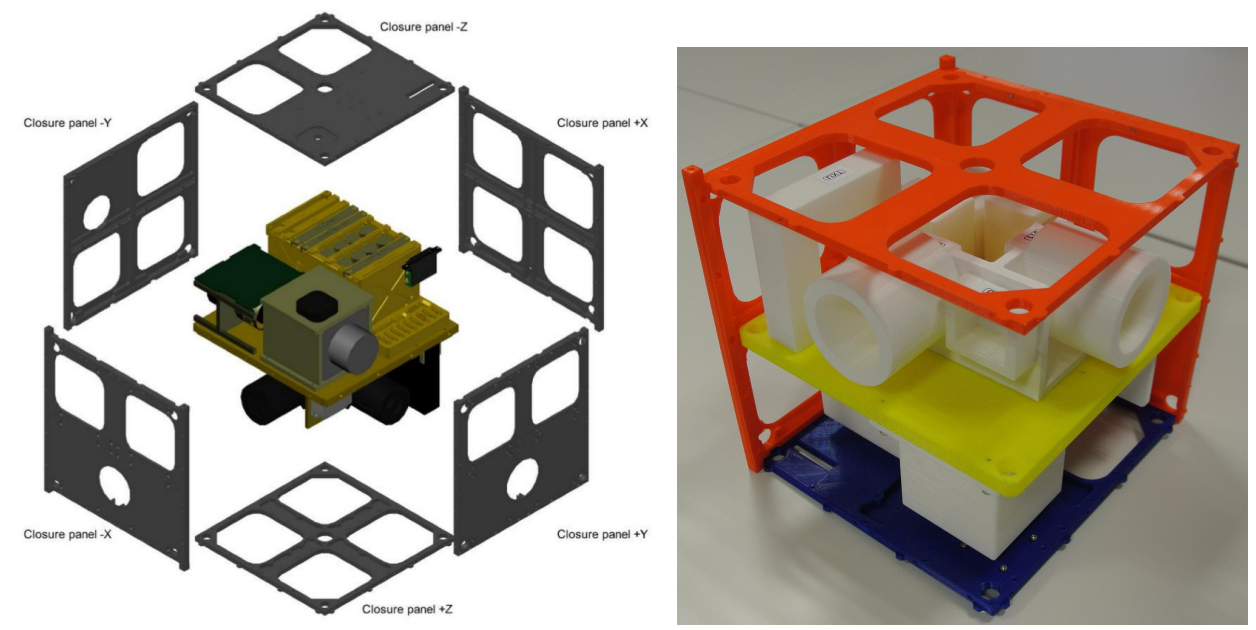

Figure 10. Left panel: RedSHIFT Spacecraft Mechanical Design Concept. Right panel: 3D printed plastic model of the test spacecraft.

Preliminary analysis and tests were already done to investigate the transition from conventional machining to 3D printing of the selected 8U CubeSat satellite (e.g., Figure 11).

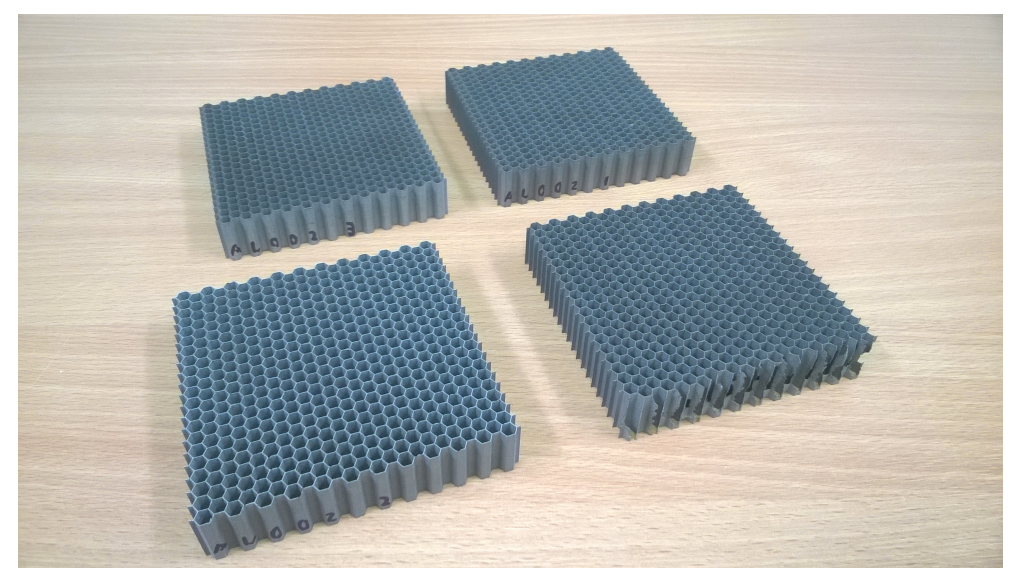

Figure 11. 3D printed samples of aluminium prototypes with 200 micron core thickness.

This constitutes a baseline study for the application of additive manufacturing to small spacecraft structures. Important manufacturing issues and "lesson learned" arose already in the first print of an $8 \mathrm{U}$ CubeSat panel oriented vertically, indicating a need to redesign any vertical structure specifically for Selective Laser Melting (SLM) production. In addition, the holes quality has been proven to be poor and not acceptable. Finally, a significant deviation of the structure was observed at the corners, which does not meet the requirements for assembly. On the basis of the test print in the subject, the following recommendations for 3D printing were derived: 
- The only feasible orientation to manufacturing the satellite panels designed for Computer Numerical Controlled (CNC) construction would be flat against the print plate. This implies ensuring that the panel geometry aligns with the specific 3D printer plate, which varies between machines and manufacturers. This also implies that none of the panels can be integrated together in the manufacturing stage.

- 3D printing allows for great design freedom and CNC milling features should be abandoned totally. As a consequence, the actions to be taken are:

- Implement mesh structure;

- $\quad$ Reduce the mass to a minimum;

- Minimize the required post-manufacturing;

- Redesign the structure.

- $\quad$ The component must be designed to minimise the amount of support structure required during the printing process. As a consequence:

- Overhang structures must be avoided;

- Sharp edges must be avoided;

- Suitable build angles must be selected.

- Holes must be avoided in the printing process and must be post-manufactured to allow the required positional precision and tolerances. As a consequence:

- Holes could be included but only with the axis of the hole aligned in the print direction, not oriented parallel to the print plate;

- Small holes must be avoided.

The preliminary results suggested a suitable 3D printing design optimization strategy, which will be explored in the next phase of the project. In particular, the design will be optimized through the following actions:

1. Additive manufacturing must be used at its potential, abandoning the idea of conventional designs suitable for subtractive manufacturing, i.e., $\mathrm{CNC}$ milling;

2. The six side panels of the Cube configuration should be simplified by using a two halves strategy, with the objective of reducing the number of fasteners, simplifying the integration process and minimizing the structural mass;

3. The Tray and Mounting bracket will be subject to a topology optimization for mass reduction, which may lead to them being merged into a single component.

We note that further design studies within the ReDSHIFT include the prototype of a 3D-printed sail attachment, presented in detail in Dalla Vedova et al. in this same issue and they therefore will not be described here.

\subsection{Shielding}

Two baseline shielding concepts were defined for 3D-printing. The shields include a multi-shock panel (MSP) (left panel of Figure 12) and a single corrugated panel (SCP). The right panel of Figure 12 shows the 3D printed prototype of one of the SCPs. The influence of key design parameters, such as overall panel thickness, bumper thickness and spacing, were considered and will be evaluated through hypervelocity impact testing. For the MSP, the default bumper thickness is set to $0.5 \mathrm{~mm}$, but values from 0.25 up to $1 \mathrm{~mm}$ were explored. The rear wall thickness in the range of $1.0 \mathrm{~mm}$ to $3.0 \mathrm{~mm}$ is realistic for most equipment, and an intermediate value of $2.0 \mathrm{~mm}$ was chosen for the first phase of investigations. Different stand-off distances between the walls in the MSPs were assumed, from $1.5 \mathrm{~cm}$ up to more than $10 \mathrm{~cm}$. 


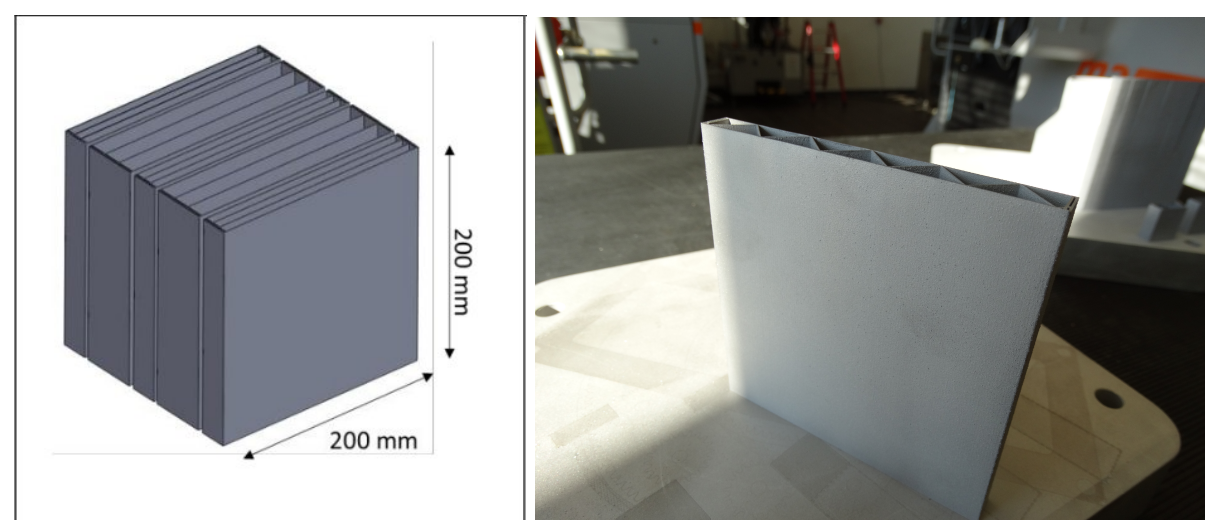

Figure 12. Left panel: set of five multi-shock panels for 3D-printing. Right panel: sample of the 3D printed SCP shield.

Two additional concepts were also anticipated, i.e., a double corrugated sandwich panel and a hybrid multi-layered panel with single corrugated bumper. These will be further investigated based on the results of the first testing phase. As a complement to this work, an impact risk analysis was performed using PHS's SHIELD3 model [12] to provide a preliminary evaluation of the shielding performance of the MSP and SCP designs. Almost 700 simulations were run to estimate the probability of penetration of each panel type when it is applied to different faces of a notional spacecraft. The designed shields appear to be suitable to protect against hypervelocity particles in the $2 \mathrm{~mm}$ to $3 \mathrm{~mm}$ size range. The simulations pointed out that the stand-off distance between the walls in the MSPs is extremely important for increased protection. Further work will be undertaken to confirm the effectiveness of the baseline shield-panels and their enhanced versions. In particular, with regard to the holistic design of ReDSHIFT, it is desirable if the shield-panels provide comparable structural and thermal performance to standard honeycomb panels whilst offering significant improvements in terms of debris shielding (and re-entry demisability).

\subsection{Testing}

A thorough theoretical and numerical analysis was preliminarily performed to identify the state of the art knowledge on the demisability of the materials and components commonly used in the manufacturing of spacecraft. The conclusion was that the current understanding of the phenomenology of the fragmentation and demise processes is limited. Therefore, it is of paramount importance to perform appropriate tests in order to improve the capability of the numerical tools to assist the design process. To this end, a set of destructive tests on spacecraft materials, structures and components (mostly 3D printed) was planned and then performed in the arc-heated supersonic wind tunnel at the German Aerospace Center (DLR). These tests included the first destructive wind tunnel tests ever performed on a complete nano-satellite and on a reaction wheel. The nano-satellite was assembled ad-hoc within the project using standard COTS components, while the reaction wheel was manufactured by Rockwell Collins.

From these tests, it has been determined that the failure of aluminium structures is highly dependent upon the behaviour of the protective metal oxide layer, and that this can be catastrophic in nature. The tests on the nano-satellite have shown that the structure can be supported by stainless steel spacers between the electronics cards, and that glass fibre reinforced plastic PCBs are more resistant to melting than had been anticipated.

Finally, the reaction wheel test has shown that the connections between parts are critical to the fragmentation and demise processes, as the glued housing separates quickly, well before melt temperature is reached at the joint. It has also demonstrated the importance of radiative cooling, as the flywheel and ball-bearing unit have survived a test at over $800 \mathrm{~kW} / \mathrm{m}^{2}$ with little damage. Figure 13 shows a few screenshots from the video of the reaction wheel test inside the DLR tunnel. 

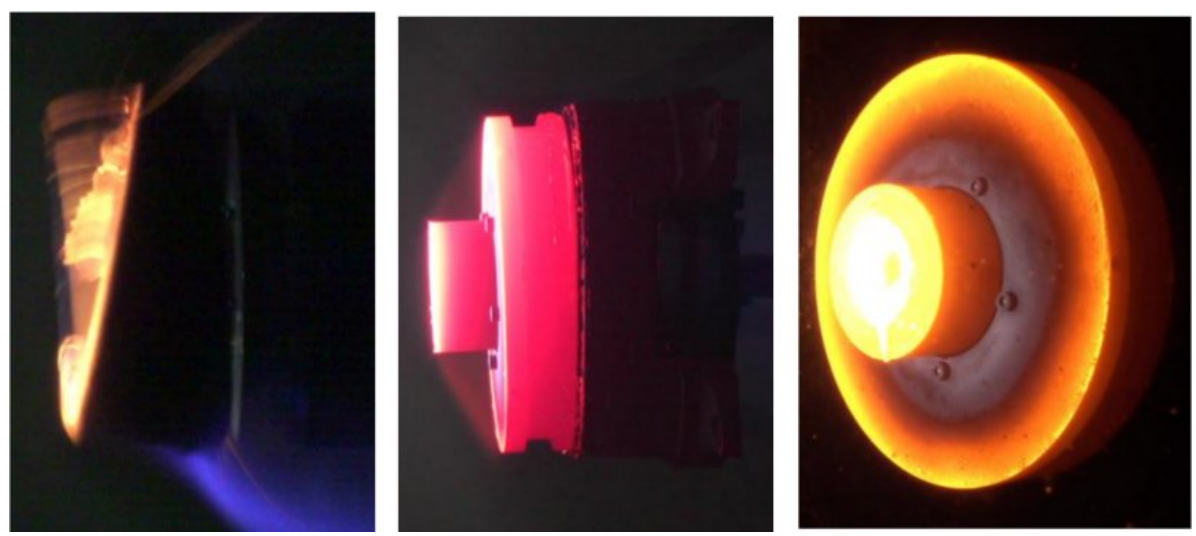

Figure 13. The reaction wheel undergoing D4D test in the DLR (German Aerospace Center) test chamber.

Further testing on the 3D printed material and the ReDSHIFT spacecraft will be performed in the coming months. These tests will include hypervelocity tests at the University of Padova light gas gun. Radiation test will be done in the Padova INFN chamber and standard vibration tests will be performed by EDSS in Spain.

\section{Software}

One of the deliverables of the ReDSHIFT H2020 project will be a software tool available to the scientific community and the public via a web-based interface. The ReDSHIFT software is thought of as a tool for spacecraft operators, space agencies and research institutions to design a space debris compliant mission, e.g., by suggesting the disposal trajectories and the technologies needed to achieve them, the best shielding opportunities for a given spacecraft and the possibility to produce it with additive manufacturing, etc. It will output safe, scalable and cost-effective satellite and mission designs in response to operational constraints.

In detail, there will be five interconnected modules:

- a disposal module (based on the results described in Section 2)

- a flux and collision probability module

- an environment projection module

- a design and shielding model (based on the results described in Section 3)

- a design for demise module (based on the results of Section 3.2).

All the modules are interfaced and linked, in a carefully triggered processing chain (Figure 14), through the openSF simulation framework properly configured and customised to adhere to the needs of the ReDSHIFT SW tool.

The core of the software is represented by the modules allowing the computation and characterization of the disposal trajectory for a given spacecraft at the end-of-life. In the disposal module, given the initial orbit of the spacecraft and the spacecraft characteristics in terms of its cross area and mass, the optimal options for end-of-life disposal are given and compared. The end-of-life disposal can be attained either via one or a sequence of impulsive manoeuvres or through the use of a solar/drag sail or through a hybrid sail and manoeuvre approach. This module is based on a study of the natural orbit evolution in the low to medium and geostationary regions that was performed to identify long-term stable orbits or resonance conditions to be used as graveyard or re-entry trajectories. The optimal manoeuvre to reach such re-entry or graveyard conditions is calculated. If the re-entry can be enhanced through a sail, different strategies for sail attitude control can be selected. The optimal disposal by this module is passed to the space environment module so that the effect of this disposal on the space debris environment is calculated. This is done based on precomputed long-term simulations 
of the whole space debris environment, under different scenarios, to be used for the computation of the collision risk for the spacecraft in the disposal phase. In the case that the disposal trajectory is a re-entry one, the conditions of the orbit at $120 \mathrm{~km}$ are used to verify the demisability of the spacecraft. This is done, by default, using some predetermined spacecraft configuration but the external user can also load a preferred configuration.

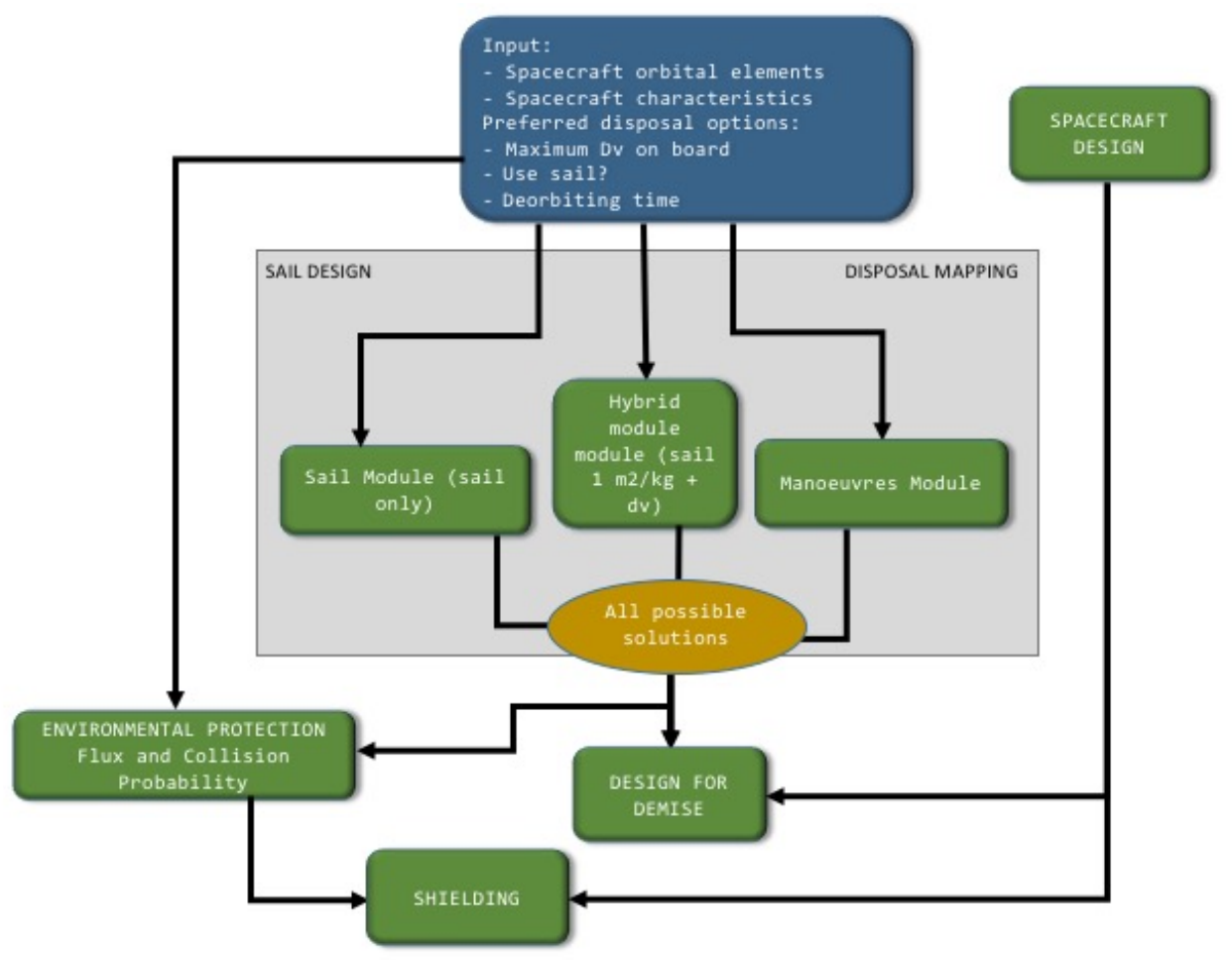

Figure 14. Schematic flow chart of the ReDSHIFT software.

\section{Legal Aspects}

The potential benefits of any proposed mitigation measure is often weakened by its limited practical application. A clear understanding of the complex legal framework of the space activities is therefore essential to try and enforce any innovative mitigation procedure. One of the problems in setting up a normative scheme for space activities in general (and space debris as an outcome of space activities in particular) is the different pace of the advancement of the technology with respect to the law, i.e., the development of new technological solutions is running much faster than the law does while the law is struggling to adapt to technological challenges. A striking example is the planning and development of the new mega-constellations of LEO satellites which are going to stress the existing guidelines to their extreme limits and might require their re-formulation or even new ones. In the framework of the ReDSHIFT project, a special effort is devoted to the study of the current international legal framework related to space debris in order to identify its deficiencies and propose possible advancements. All international space debris mitigation instruments so far have a non-binding character and rely on voluntary adherence. Nevertheless, they provide detailed and practical guidelines (e.g., [8]). They may acquire a binding character provided that there is enough uniform practice which evolves to customary law. Moreover, these guidelines also play a role by serving as a basis for national laws (such as the French and Austrian space law). Two major problems can be immediately identified in the preliminary analysis of the existing national laws: there is no uniformity of national standards (e.g., different definitions of protected regions in LEO, MEO and GEO) and there appears different waivers with justification, for example for small satellites. In the 
analysis of an optimised legal and political framework able to better promote the application of improved guidelines, it is important to explore how the new design and technologies and rules foster specific branches of the economy. In the environmental economy, there are clear evidences that, contrary to common feelings and initial expectations, new rules are often fostering innovation, urging manufacturers to move from the status quo towards new, often economically profitable technical solutions. The aim of the legal/political and economical analysis within ReDSHIFT is also to push the space manufacturers towards these innovative and possibly profitable directions. Therefore, next to legal mechanisms, economic incentives could also contribute to the 'attractiveness' of the adherence to mitigation standards if properly coupled with viable novel technical solutions. Following this strategy, a few examples of the proposed measures under study include:

- the possibility to exploit the newly identified re-entry "corridors", steering the space traffic towards these preferential routes for disposal, following the extensive cartography of the circumterrestrial phase space, as explained in Section 2;

- the re-definition of the concept of residual lifetime implicit in the 25-year rule, towards a concept considering the actual interaction of a spacecraft with a given protected zone;

- the possibility to apply economic incentives to such promotion of deorbiting devices (similar to what is sometimes seen on Earth in eco-friendly devices and transportation means).

\section{Conclusions}

After more than two years of work, the H2020 ReDSHIFT project has already reached a number of interesting goals which were briefly summarized in the above sections. In particular, we can highlight:

- a complete mapping of the LEO to GEO space was performed and the cartography was exploited to devise "dynamical" disposal strategies for any orbital regime.

- a prototype small spacecraft "debris compliant" was designed and assembled, exploiting the advantages offered by the additive manufacturing procedures. Several parts, including optimized debris shields, were designed and 3D printed.

- the materials and components of the spacecraft were tested for Design for Demise (D4D). Moreover the D4D tests included a novel test on an engineering model of a reaction wheel.

- the possibility to exploit area augmentation devices (e.g., solar and drag sails) was studied both from the dynamical and the hardware point of view.

- a software tool (whose web version will be made public at the end of the project) encompassing all the above findings was produced. The software shall help the users to conceive a "debris compliant" space mission from the design to the disposal phase.

- the possible improvements to the international space regulations and standards, stemming from the project's findings, were analyzed.

Author Contributions: A.R. is the coordinator of ReDSHIFT and the main writer of this document. A.R., C.C., K.T., V.S., E.M.A., G.S., D.K.S., I.G., F.B.Z., E.S. contributed mainly to the results in Sections 2 and 4. J.B., I.H. and T.S. contributed mainly to the results in Section 3.2. J.B.R., S.W., F.D.V., J.B. and I.H. contributed to the results in Section 3. A.F. and H.S. contributed to the results in Section 3.1. F.L. contributed to the results in Section 4. R.P. and Y.K. contributed to the results in Section 5.

Acknowledgments: The research leading to these results has received funding from the Horizon 2020 European Unions Framework Programme for Research and Innovation (H2020-PROTEC-2015) under REA grant agreement n. [687500]-ReDSHIFT. The authors also express their gratitude to the ESA/ESOC Space Debris Office for granting permission to use the MASTER 2013 reference population in the context of ReDSHIFT.

Conflicts of Interest: The authors declare no conflict of interest. 


\section{References}

1. Schaus, V.; Radtke, J.; Stoll, E.; Rossi, A.; Colombo, C.; Tonetti, S.; Holbrough, I. Results of reference long-term simulations focussing on passive means to reduce the impact of space debris. In Proceedings of the 7th European Conference on Space Debris, Darmstadt, Germany, 18-21 April 2017.

2. Alessi, E.M.; Schettino, G.; Rossi, A.; Valsecchi, G.B. Natural Highways for End-of-Life Solutions in the Region. Celest. Mech. Dyn. Astron. 2018, 130, 34. [CrossRef]

3. Colombo, C.; Gkolias, I. Analysis of orbit stability in the geosynchronous region for end-of-life disposal. In Proceedings of the 7th European Conference on Space Debris, Darmstadt, Germany, 18-21 April 2017.

4. Rosengren, A.; Skoulidou, D.; Tsiganis, K.; Voyatzis, G. Dynamical cartography of Earth satellite orbits. Adv. Space Res. 2018, submitted.

5. Skoulidou, D.K.; Rosengren, A.J.; Tsiganis, K.; Voyatzis, G. Cartographic study of the MEO phase space. In Proceedings of the 7th European Conference on Space Debris, Darmstadt, Germany, 18-21 April 2017.

6. Skoulidou, D.K.; Rosengren, A.J.; Tsiganis, K.; Voyatzis, G. Dynamical Lifetime Survey of Geostationary Transfer Orbits. Celest. Mech. Dyn. Astron. 2018, submitted.

7. Alessi, E.M.; Schettino, G.; Rossi, A.; Valsecchi, G.B. Solar radiation pressure resonances in Low Earth Orbits. Mon. Not. R. Astron. Soc. 2018, 473, 2407-2414. [CrossRef]

8. Inter-Agency Space Debris Coordination Committee (IADC). IADC Space Debris Mitigation Guidelines; IADC-02-01; IADC: 2007; Revision 1. Available online: https:/ / www.iadc-online.org/Documents/ (accessed on 1 February 2018).

9. Schettino, G.; Alessi, E.M.; Rossi, A.; Valsecchi, G.B. Exploiting dynamical perturbations for the end-of-life disposal of spacecraft in LEO. Acta Astronaut. 2018, submitted.

10. Gkolias, I.; Colombo, C. End-of-life disposal of geosynchronous satellites, Paper IAC-17-A6.4.3. In Proceedings of the 68th International Astronautical Congress, Adelaide, Australia, 25-29 September 2017.

11. Colombo, C.; de Bras de Fe, T. Assessment of Passive and Active Solar Sailing Strategies for End-of-Life Re-entry, Paper IAC-16-A6.4.4. In Proceedings of the 67th International Astronautical Congress, Guadalajara, Mexico, 26-30 September 2016.

12. Stokes, H.; Cougnet, C.; David, M.; Gelhaus, J.; Röthlingshöfer, M. A detailed impact risk assessment of possible protection enhancements to two LEO spacecraft. In Proceedings of the 6th European Conference on Space Debris (ESOC), Darmstadt, Germany, 22-25 April 2013.

(C) 2018 by the authors. Licensee MDPI, Basel, Switzerland. This article is an open access article distributed under the terms and conditions of the Creative Commons Attribution (CC BY) license (http:/ / creativecommons.org/licenses/by/4.0/). 